\title{
A comparative study of albumin-bilirubin score, MELD and Child Pugh scores for predicting the in-hospital mortality in cirrhotic patients complicated with Upper GI bleeding in a Tertiary care hospital
}

\author{
Nagaraja B S', Madhumathi R², Umesh K J33, Rashwith $\mathbf{U}^{4}$ \\ ${ }^{1}$ Professor and Head, ${ }^{2}$ Professor, ${ }^{3,4}$ Post graduate, Department of Medicine, Bangalore Medical College and Research \\ Institute, KR road, Bangalore, Karnataka, India
}

A B S T R A C T

Background: Alcoholic liver Disease (ALD) encompasses a spectrum of Injury, ranging from simple steatosis to frank cirrhosis which is evaluated by many scoring systems. Aims and Objective: Our study aims at evaluating the discriminative abilities of Child-Pugh, model for end-stage liver disease (MELD), and albumin-bilirubin (ALBI) scores in predicting the inhospital mortality in cirrhotic patients complicated with acute upper gastrointestinal bleeding. Materials and Methods: Data of Patients with Liver cirrhosis secondary to ethanol presented with Upper GI bleed were retrospectively reviewed. Child Pugh, MELD and ALBI scores were calculated for the patients and results from ROC curves were analysed. Results: In our study conducted on 112 patients, the age distribution was between 18-74 yrs with mean age of patients being $46.47+/-10.9$ years, sex ratio Male: Female: 105:7 with mortality rate of $33.4 \%$.the Area under curves of ROC of ALBI, Child Pugh and MELD are 0.743, 0.864 and 0.763. Conclusion: The prognostic performance of all 3 scores was comparable but Child Pugh score was found to have better prognostic significance than ALBI and MELD score.

\section{Access this article online}

Website:

http://nepjol.info/index.php/AJMS DOI: 10.3126/ajms.v10i1.20748 E-ISSN: 2091-0576

P-ISSN: 2467-9100

Key words: Albumin Bilirubin score; Acute Upper Gastrointestinal bleed; Model for end stage liver disease

\section{INTRODUCTION}

Alcoholic liver Disease (ALD) encompasses a spectrum of Injury, ranging from simple steatosis to frank cirrhosis. ${ }^{1}$ Alcohol related liver disease is the commonest cause of death, accounting for 2.5 million/yr. ${ }^{2}$ Upper Gastro Intestinal Bleeding related to portal hypertension is a lethal complication in patients with liver cirrhosis. Child Pugh and Meld scores are two of the most important models for predicting the survival of acute upper Gastro intestinal Bleeding in cirrhotic patients. ${ }^{3,4}$ Recently the Albumin Bilirubin score (ALBI) has been established as a convenient and evidenced based model to access the severity of liver dysfunction in patients with hepatocellular carcinoma(HCC). ${ }^{5}$
ALBI Score (Albumin-Bilirubin Score) is a new mathematical model for evaluation of liver function. It is calculated by using the formula $-0.085 \times$ (albumin $\mathrm{g} / \mathrm{l}$ ) $+0.66 \times \log$ (bilirubin $\mu \mathrm{mol} / \mathrm{l}$ ). It is a mathematical formula using only two parameters, based on routine blood tests, avoids subjective assessment of ascites and encephalopathy and without arbitrary cut-offs as compared to child Pugh score. ${ }^{6}$

However, the role of the ALBI score for the assessment of prognosis of AUGIB in liver cirrhosis remains unclear. Herein we conducted a retrospective study in cirrhotic patients with Upper GI Bleed to compare the prognostic performance of the ALBI score with CHILD PUGH and MELD scores. 
Aims and objectives

1. To calculate Albumin Bilirubin score, Child Pugh score and MELD scores for cirrhotic patients complicated with upper Gastro intestinal bleeding.

2. To evaluate the discriminative abilities of ChildPugh, model for end-stage liver disease (MELD), and albumin-bilirubin (ALBI) scores in predicting the in-hospital mortality in cirrhotic patients with acute upper gastrointestinal bleeding.

\section{MATERIALS AND METHODS}

\section{Study design}

The study was conducted at Bowring and Lady Curzon Hospital (Attached to Bangalore Medical College and Research institute). Cirrhotic patients secondary to ethanol complicated with acute upper GI bleed admitted in the Hospital between 2016 January and 2016 December were retrospectively reviewed and the data of the patients were collected.

\section{Inclusion criteria}

1) Patient aged $>18$ years.

2) Cirrhotic patients with Upper gastro intestinal Bleeding.

\section{Exclusion criteria}

1) Chronic liver disease due to hepatitis B, C, malignancy, metabolic causes, autoimmune hepatitis, Non variceal bleed patients.

\section{Method of collection of data}

Detailed history and clinical Examination done for all the patients were noted from the case sheets. Routine investigations like CBP, RFT, LFT, serum electrolytes, HIV, HBsAg, HCV, VDRL serology, prothrombin time, APT'T, Ultrasound of abdomen, upper GI endoscopy and other relevant investigations were noted. Diagnosis of liver cirrhosis was established by USG abdomen with shrunken liver with altered echo texture.

Complications like anemia, hepatic encephalopathy, renal dysfunction and mortality secondary to upper GI bleed were noted.

ALBI $(-0.085 \times$ (albumin $\mathrm{g} / \mathrm{l})+0.66 \times \log$ (bilirubin $\mu \mathrm{mol} / \mathrm{l}))$, Child-Pugh and MELD $(10 *((0.957$ $* \ln ($ Creatinine $))+(0.378 * \ln ($ Bilirubin $))+(1.12 *$ $\ln (\mathrm{INR})))+6.43$ scores were calculated and compared.

\section{Method of statistical analysis}

All statistical analysis were performed using the Medcalc software.Continuous Data were expressed as the mean $+/$ - standard deviation(SD) and median with minimum and maximum. Categorical data were expressed as the frequency. Receiving-operative characteristics curve analysis was performed to identify the discriminative ability of the ALBI, Child Pugh and MELD scores in predicting in-hospital mortality. Areas under the ROC curves were calculated and compared. The best cut off value was selected as the sum of sensitivity and specificity was maximal. Then sensitivity, specificity, positive likelihood ratio, negative likelihood ratio were reported.

\section{RESULTS}

The sample size in our study was 112 patients. The age distribution was between 18-74 yrs, with mean age of patients being $46.47+/-10.9$ years (Table 1 ). One hundred and five (105) were males and Seven (7) were females(Table 2).

\begin{tabular}{lcc}
\multicolumn{3}{l}{ Table 1: Age distribution of patients studied } \\
\hline Age in years & No. of patients & $\%$ \\
\hline $20-30$ & 9 & 8.0 \\
$31-40$ & 26 & 23.2 \\
$41-50$ & 47 & 42.0 \\
$51-60$ & 21 & 18.8 \\
$61-70$ & 8 & 7.1 \\
$>70$ & 1 & 0.9 \\
Total & 112 & 100.0 \\
\hline Mean \pm SD: $46.47 \pm 10.90$ & &
\end{tabular}

\begin{tabular}{lcc} 
Table 2: Gender distribution of patients studied \\
\hline Gender & No. of patients & $\%$ \\
\hline Female & 7 & 6.3 \\
Male & 105 & 93.8 \\
Total & 112 & 100.0 \\
\hline
\end{tabular}

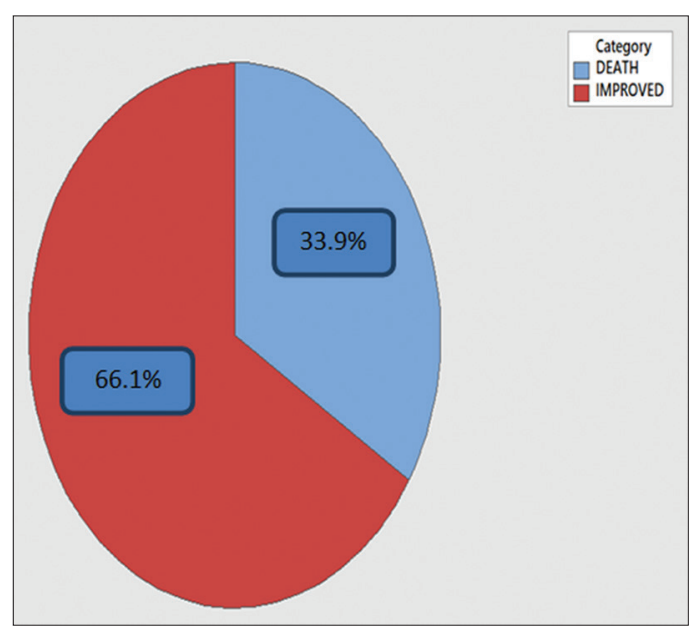

Figure 1: Pie chart: Death: 38 Improved: 74 
Among 112 patients reviewed, 38 were deaths and 74 were patients who showed improvement, with mortality percentage of $33.4 \%$ (Figure 1).

Comparison of in-hospital mortality with ALBI, Child PUGH and MELD scores

The in Hospital morality was $33.9 \%$. The Area under curve (AUC) of the ALBI score for predicting the inhospital mortality was 0.743 (confidence interval: 95\%: $0.652-0.821$ ). The best cut-off value of -0.743 , with sensitivity of $89 \%$, a specificity of $52.7 \%$, positive likelihood ratio(PLR) of 1.255 and negative likelihood ratio (NLR) 0.23 .

The AUC of the Child Pugh score for predicting the in-hospital mortality was 0.864 (confidence interval $95 \%$ : 0.786-0.921). The Best cut-off value of the Child-Pugh score was 12 , with a sensitivity of $84.2 \%$, a specificity of $75.6 \%$, PLR of 3.62 and NLR of 0.2 .

The AUC of the MELD score for predicting the in-hospital mortality was 0.762 (confidence interval $95 \% 0.672-0.837$ ). The Best cut-off of the MELD score was 25.5, with a sensitivity of $73.7 \%$, a specificity of $69.9 \%$, PLR of 3.49 and NLR of 0.381 .

The AUC for predicting the in-hospital mortality was significantly different between the Child Pugh versus ALBI and MELD scores.(Child Pugh and ALBI: $\mathrm{P}=0.0193$; ChildPugh and MELD: $\mathrm{P}=0.0397$ )

Order of sequence in respect to AUC: CHILD-PUGH $>$ MELD > ALBI

ROC curve of child pugh score for predicting in hospital mortality

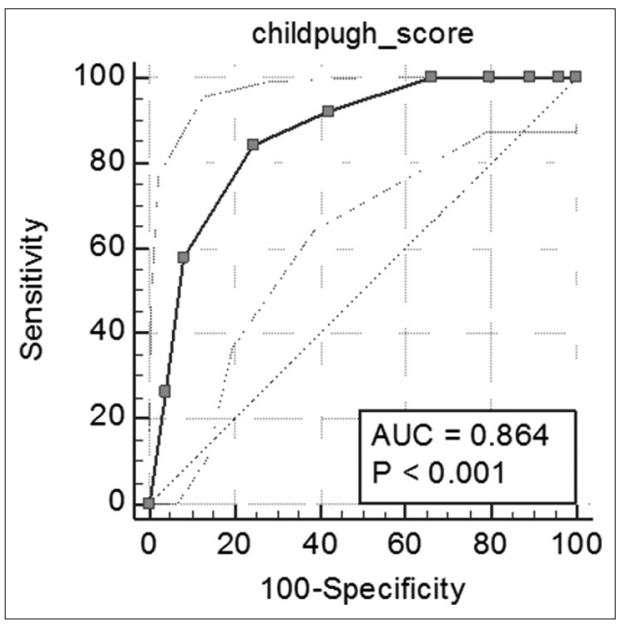

ROC curve of ALBI score for predicting In Hospital Mortality

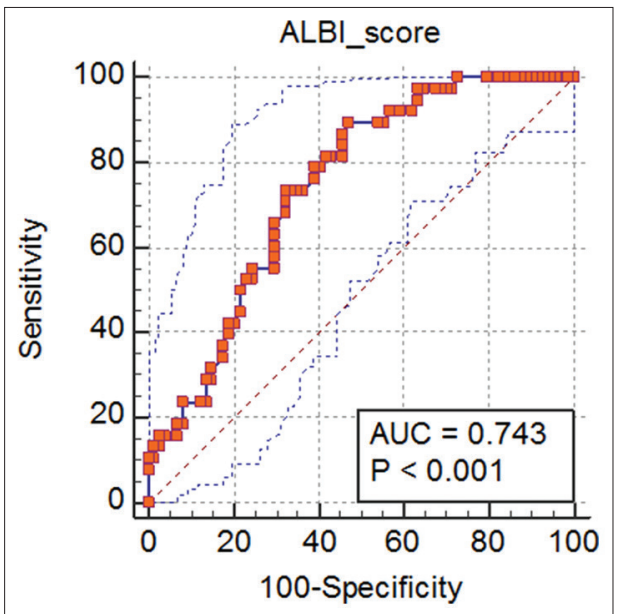

ROC curve of MELD score for predicting in hospital mortality
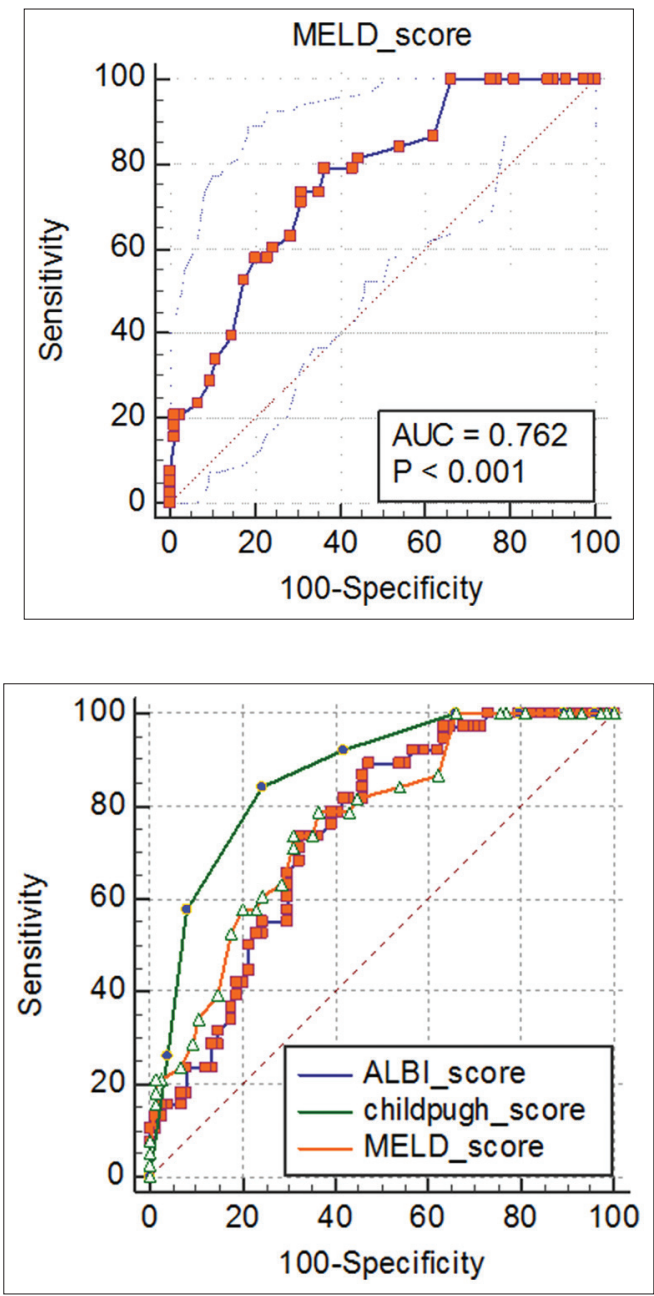
Comparison of all 3 scores

\begin{tabular}{lccc}
\hline Variable & AUC & SE $^{\mathrm{a}}$ & $\mathbf{9 5 \%} \mathbf{C l}^{\mathbf{b}}$ \\
\hline ALBI_score & 0.743 & 0.0461 & 0.652 to 0.821 \\
childpugh_score & 0.864 & 0.0340 & 0.786 to 0.921 \\
MELD_score & 0.762 & 0.0457 & 0.672 to 0.837 \\
\hline
\end{tabular}

\section{DISCUSSION}

The First appearance and subsequent growth of gastrooesophageal varices following diagnosis of varices is approximately $7 \%$ per year. ${ }^{7,8}$ Degree of the liver dysfunction is the best predictor of variceal bleeding. ${ }^{9}$ It is well known that there are several scoring systems available to evaluate the severity of liver dysfunction and predict the prognosis of patients with liver Disease, such as the MELD and Child- Pugh score scores.

The Child- Pugh Score contains Five Parameters, including the Total Bilirubin, Serum Albumin, Prothrombin time, ascites and Hepatic Encephalopathy. However the Highly Subjective evaluation of Ascites and encephalopathy might reduce the accuracy of assessment. ${ }^{8}$

The MELD score incorporates 3 laboratory variables, Total Bilirubin, INR and creatinine, and it eliminates the subjective factors. ${ }^{8}$ The MELD score was widely used as a scoring system for organ allocation in liver Transplantation and is the current standard prognostic tool for assessing the 3 to 6 month survival in patients with Failure. ${ }^{11}$

The ALBI score involves only 2 common laboratory parameters, albumin and total bilirubin and it is being used in patients with Hepatocellular carcinoma for accessing the severity of Liver dysfunction. ${ }^{12}$

In our study, we tried to explore the prognostic performance of the ALBI score for the assessment of the in-hospital mortality of AUGIB in liver cirrhosis. We found that the prognostic performance of the ALBI score was comparable to that of Child Pugh and MELD scores.

In overall analysis, Child Pugh score had the largest AUC, followed by MELD and ALBI scores. So according to our study, Child Pugh score has better prognostic performance compared to ALBI and MELD score.

In a study done by Lichun Sao et al, department of gastro enterology 1067 cirrhotic patients were retrospectively enrolled and receiver operating characteristic curve analyses was done. Area under curves of the study was in following order: child pugh > MELD > ALBI. Later concluded that ALBI score might be an alternative index for assessing the in-hospital mortality in patients with liver cirrhosis. ${ }^{13}$
In a similar study conducted by Deli Zou et al, department of gastroenterology in 2016 on 631 patients, ALBI score had the largest AUC, followed by MELD score and Child Pugh scores in ROC curves, so they concluded that ALBI score has moderate to high prognostic performance. ${ }^{14}$

A retrospective study done by Xavier SA et al on 111 patients between Jan 2011 and Nov 2015, came out with conclusion that ALBI score is particularly useful in the assessment of short come outcomes, with a better performance than the most commonly used scores. Area under curves (of ROC) were ALBI- 0.75, child Pugh- 0.64, and MELD $-0.72 .^{15}$

A study conducted by Peng et al showed there was no significant difference in predicting in-hospital mortality in cirrhotic patients. Area under curves (of ROC) was ALBI- 0.57, child Pugh- 0.59, and MELD -0.57. ${ }^{16}$

A study conducted by Flores-Rendon AR et al in 2008, on 212 cirrhotic patients with variceal bleeding concluded that MELD score was significantly better than Child-Pugh score for predicting in hospital mortality related to variceal bleeding.(AUC were MELD: 0.905 and child-Pugh 0.794). ${ }^{17}$

\section{CONCLUSION}

Child Pugh score has the Largest AUC, followed by MELD and ALBI scores indicating that CHILD-PUGH scorehas better Prognostic significance when compared to ALBI and MELD score.So, Child Pugh score being calculated easily (compared to other scores) and accounting for more parameters has the better prognostic performance compared to ALBI and MELD score, Hence can be continued to be used in peripheral centres to assess the prognosis of chronic liver disease patients presenting with complications like Upper GI Bleed.

\section{Limitations of the study}

1) Long term follow up was unavailable, so this study couldn't evaluate the role of ALBI for predicting LONG term Prognosis.

2) Mortality was very high, because the mean Child Pugh score of presenting patients was 12 .

3) ALBI scores are not dynamically measured, therefore whether the albi score is step wise elevation based on the progressive deterioration of liver function remains unclear.

\section{REFERENCES}

1. O'Shea RS, Dasarathy $S$ and McCullough AJ. Alcoholic Liver Disease, The American Journal of Gastroenterology 2010;105(1):14-32. 
2. Mark E Mailliard, Micheal F Sorell. Alcoholic Liver Disease. Harrison's Principles of Internal Medicine. $19^{\text {th }}$ ed. New York. McGraw Hill; 2015:p2052.

3. Child CG and Turcotte JG. Surgery and portal Hypertension. Major problemin Clinical Surgery 1964: 1: 1-85.

4. Kamath PS and Kim WR. Advanced Liver Disease Study group, The Model for end stage liver disease(MELD). Hepatology 2007; 45(3):797-805.

5. Knox JJ.Addressing the interplay of liver Disease and Hepatocellular Carcinoma on Patient Survival: the ALBI scoring model. Journal of Clinical Oncology 2015;33(6):529-531.

6. Goldis A, Lupusoru R, Goldis R and Ratis L. Prognostic Factors in Liver Cirrhosis Patients with upper gastrointestinal Bleeding. Biology and Medicine (Aligarh. 2017;10(1):423.

7. GroszmannRJ, Garcia-Tsao G, Bosch J, Grace ND, Burrough's AK, Planas R, et al, Beta blockers to prevent gastro oesophageal varices in patients with cirrhosis. The New England Journal of Medicine 2005; 353: 2254-2261.

8. Merli M, Nlcolini G, Angeloni S, Rinaldi V, De Santis A, Merkel, et al. Incidence and Natural history of small oesophageal varices in cirrhotic patients.Journal of Hepatology 2003; 38: 266-272.

9. North Italian Endoscopic Club For study and treatment of Oesophageal varices, Prediction of the First variceal Haemorrhage in Patients with cirrhosis of the liver and oesophageal varices. A prospective Multicentre Study The New England Journal Medicine, 1988; 319(15): 983-989.

10. Kamath PS, Weisner RH, Malinchoc M, Kremers W, Therneau TM, Kusberg CL, et al, A Model to predict survival in patients with end stage liver disease. Journal of Hepatology $2001 ; 33: 464-470$
11. Freeman RB, Weisner RH, Harper A, Mcdiarmid SV, Lake J, Edwards $E$, et al. The new liver allocation System: moving toward evidence based transplantation policy. Journal of liver Transplantation 2002;8(9):851-858.

12. Johnson PJ, Berhane S, Kagebayashi C, Satomura S, Teng M, Reeves $\mathrm{HL}$, et al. Assessment of liver function in patients with hepatocellular carcinoma: a new evidence based approach- The ALBI grade. The Journal of Clinical Oncology 2015; 33(6):550-558.

13. Shao L, Han B, Shu AN, Jiaxin Ma, Xiaohong Guo, Romeiro FG, et al. Albumin-to-Bilirubin Score for Assessing the in-Hospital Death in Cirrhosis. Transl Gastroenterol Hepatol 2017; 2:88.

14. Zou D, Qi X, Zhu C, Ning Z, Hou F, Zhao J, et al. Albumin-bilirubin score for predicting the in-hospital mortality of acute upper gastrointestinal bleeding in liver cirrhosis: A retrospective study. The Turkish Journal of Gastroenterology 2016; 27(2):180-186.

15. Xavier SA, Vilas Boas R, Boal Carvalho $P$, Magalhaes JT, Marinho CM and Cotter JB. Assessment of prognostic performance of Albumin Bilirubin, Child Pugh and Model for End stage Liver disease scores in patients complicated with Acute upper gastrointestinal bleeding. The European Journal of Gastroenterology and Hepatology 2018; 30(6):652-658.

16. Peng Y, Qi X, Tang S, Deng H, Li J, Ning Z, et al. Child-Pugh, MELD, and ALBI scores for predicting the in-hospital mortality in cirrhotic patients with acute-on-chronic liver failure. Expert Rev Gastroenterol Hepatol 2016; 10:971-980.

17. Flores-Rendon AR, Gonzalez-Gonzalez JA, Garcia-Compean D, Maldonado-Garza-HJ and Garza-Galindo-AA. Model for end stage of liver disease (MELD. is better than the Child-Pugh score for predicting in-hospital mortality related to esophageal variceal bleeding. Annals of Hepatology 2008; 7(3):230-234.

\footnotetext{
Authors Contribution:

NBS- Concept and Design of the study, Manuscript preparation, Statistically analysed and interpreted, critical revision of the manuscript; MR- Concept and Design of the study,Statistically analysed and interpreted,critical revision of the manuscript; UKJ- reviewed the literature, helped in preparing first draft of Manuscript, collected data; RU- collected data, Statistically analysed and interpreted, helped in preparing draft of manuscript.

Work attributed to: Department of General Medicine, Hospitals attached to Bangalore Medical College and Research institute.

Orcid ID:

Dr.Nagaraja B S - (i) https://orcid.org/0000-0002-7750-697X

Dr. Madhumathi R - (D https://orcid.org/0000-0002-7534-1059

Dr. Umesh K J - (D) https://orcid.org/0000-0002-1816-9125

Dr.Rashwith U - (1) https://orcid.org/0000-0002-7369-1645

Source of Support: Bangalore Medical College and Research Institute fund, Conflict of Interest: None.
} 